\title{
El Rendimiento Académico de Estudiantes de Odontología en la Asignatura de Morfología como Predictor del Tiempo Real de Titulación y Rendimiento Académico en Asignaturas de Alto Crédito
}

\author{
Academic Performance of Dental Students in the Subject of Morphology as a Predictor \\ of their Actual Graduation Time and Academic Performance in High Credit Subjects
}

Melisa Grob ${ }^{1}$; César Orsini²; Daniela Becerra ${ }^{1}$; Natividad Sabag ${ }^{1} \&$ María Paz Rojas $^{1}$

GROB, M.; ORSINI, C.; BECERRA, D.; SABAG, N. \& ROJAS, M. P. El rendimiento académico de estudiantes de odontología en la asignatura de morfología como predictor del tiempo real de titulación y rendimiento académico en asignaturas de alto crédito. Int. J. Morphol., 38(5):1508-1512, 2020.

RESUMEN: La relevancia de las ciencias básicas como predictor de rendimiento académico presenta gran importancia en el área de la salud. Por lo tanto, el objetivo de este estudio fue analizar el rol predictivo del desempeño de los estudiantes de Odontología en la asignatura de Morfología sobre las asignaturas de alto crédito en la fase preclínica y clínica, y sobre el tiempo real de titulación. Se llevó a cabo un estudio correlacional retrospectivo, con 672 estudiantes de Odontología (2002 a 2012) de la Universidad de los Andes, Chile. Las variables estudiadas fueron sexo, año de ingreso y de titulación, y rendimiento académico en las asignaturas de Morfología (primer año), Preclínico del Adulto (tercer año), y Clínica Integral del Adulto de cuarto y quinto año. El rol predictivo se analizó mediante pruebas de correlaciones de Pearson y análisis de ecuaciones estructurales. Se observó que el rendimiento académico de Morfología se asocia y predice de forma significativamente positiva el rendimiento académico en Preclínico del Adulto $(\mathrm{r}=0,25 \mathrm{p}<, 01, \mathrm{~b}=0,250$ $\mathrm{p}<, 001)$, Clínica del Adulto de cuarto año $(r=0,20 \mathrm{p}<, 01, \mathrm{~b}=0,198 \mathrm{p}<, 001)$ y Clínica del Adulto de quinto año $(\mathrm{r}=0,14 \mathrm{p}<, 01, \mathrm{~b}=0,138$ $\mathrm{p}<, 001)$, y se asoció y predijo de forma significativamente negativa el tiempo real de Titulación ( $\mathrm{r}=-0,48 \mathrm{p}<, 01, \mathrm{~b}=-0,258 \mathrm{p}<, 001)$. El modelo de rendimiento académico de la asignatura de Morfología y su influencia sobre las otras asignaturas explicó un 68,5\% de varianza en el tiempo real de titulación. Los resultados de este estudio contribuyen a robustecer la evidencia disponible en relación a la relevancia del estudio y buen desempeño en Morfología para el desarrollo y formación de los estudiantes a lo largo del currículo Odontológico, además de justificar la identificación oportuna y apoyo para aquellos estudiantes que estén experimentando dificultades en esta asignatura. titulación.

PALABRAS CLAVE: Educación superior; Morfología; Odontología; Sistema de créditos transferibles; Tiempo real de

\section{INTRODUCCIÓN}

La relevancia de las ciencias básicas y del estudio de la morfología para el currículo odontológico se ve respaldada por gran número de asociaciones internacionales, las cuales han establecido al estudio de la morfología como un aspecto indispensable para el diseño curricular (Australian Dental Council, 2016; Field et al., 2017; American Dental Education Association, 2017). Estos estándares destacan a la integración curricular de tipo vertical como clave, entendida como una secuencia de organización curricular en la cual se integra a la morfología con las disciplinas preclínicas y clínicas (Hassan, 2013). De esta forma, el valor de las ciencias básicas ha tomado gran relevancia para el desarrollo y predicción profesional de los Odontólogos (Fiehn, 1998; Martínez-Alvarez et al., 2001; Bennett \& Beeley, 2013).
Tradicionalmente, el currículo en Odontología comprende 6 años dividido en una fase inicial de ciencias básicas, una fase preclínica abarcando parte del segundo año y el tercer año, y finalmente una fase clínica que abarca desde parte del tercer año al sexto año (Plasschaert et al., 2006; Serrano et al., 2018). Existe un número limitado de estudios que evalúa el rol predictor de las ciencias básicas sobre el desempeño de los estudiantes a lo largo de currículo (Rodríguez Fontes et al., 2000; Woods et al., 2005; Zúñiga et al., 2009).

En Odontología se ha reportado una correlación positiva entre los criterios de selección para ingresar al plan de estudio y el rendimiento académico en la asignatura de Mor-

\footnotetext{
${ }^{1}$ Unidad de Morfología, Facultad de Odontología, Universidad de los Andes, Santiago, Chile.

2 Área de Formación Docente, Facultad de Odontología, Universidad de los Andes, Santiago, Chile.
} 
fología (RAM) (Grob et al., 2015), por otro lado, no se reportan evidencias sobre cómo el desempeño en las ciencias básicas influye sobre otros criterios de desempeño de los estudiantes a lo largo del currículo. Entre estos criterios en el contexto de Educación Superior en Chile, además del desempeño en asignaturas de alto crédito, se ha identificado al tiempo real de titulación (TRT) como un aspecto a ser considerado al momento de evaluar un programa de estudios (Jerez et al., 2018).

Por lo tanto, el objetivo de este estudio fue analizar el rol predictivo del desempeño de los estudiantes de Odontología en Morfología, asignatura clave y de alto crédito en el ciclo de ciencias básicas, sobre otras asignaturas clave y de alto crédito en la fase preclínica y clínica, y sobre el TRT.

Hipótesis evaluadas en el estudio (Fig. 1).

- El RAM predice de forma positiva el rendimiento académico en las asignaturas de Preclínico del Adulto (PA) de tercer año, Clínica Integrada del Adulto de cuarto año (CIA4), y Clínica Integrada del Adulto de quinto año (CIA5). A su vez, el rendimiento académico en estas asignaturas predicen de forma negativa el TRT.

- El RAM predice de forma negativa el TRT.

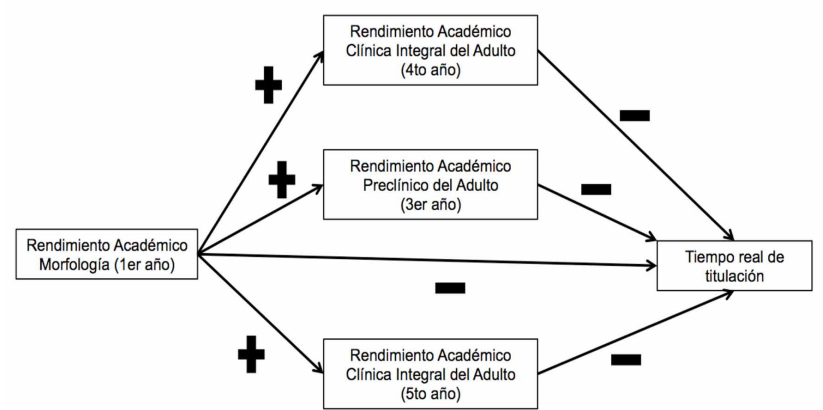

Fig. 1. Hipótesis de modelo predictivo entre rendimiento académico en la asignatura de Morfología y las asignaturas de Preclínico y Clínica Integral del Adulto, y el tiempo de titulación real. Nota: Los símbolos representan la dirección de la predicción, positiva o negativa.

\section{MATERIAL Y MÉTODO}

La investigación se llevó a cabo durante el año 2018 con un diseño correlacional retrospectivo, en el cual se analizó la información registrada sobre estudiantes de Odontología en las cohortes de ingreso entre los años 2002 y 2012 de la Universidad de los Andes, Chile. El protocolo de investigación fue aprobado por el Comité de Ética de la Universidad de los Andes (CEC201847).
Recolección de datos y variables a utilizar. Se accedió a la información desde la Unidad de registro académico de la Facultad de Odontología, con una planilla anónima que registra datos de todos los alumnos para las cohortes de ingreso entre los años 2002 y 2012 con respecto a: sexo, año de ingreso y de titulación, RAM (primer año), PA (tercer año), y CIA4 y CIA5.

La planilla no permitió la identificación de los estudiantes por parte de los autores, manteniendo así la confidencialidad de la información. Las cohortes de estudio se situaron entre los años 2002 y 2012 debido a que representan la primera generación de ingreso y el último año de ingreso en completar los 6 años de estudio formal requeridos para la titulación al momento de realizar el estudio. Con la información de año de ingreso y titulación se generó la variable de TRT.

Además de obtener información sobre el RAM, se seleccionaron las asignaturas de PA y CIA4 y CIA5 debido a que son consideradas las asignaturas claves del currículo con el mayor número de créditos. Las asignaturas de Morfología (primer año), PA (tercer año), y CIA4 y CIA5, corresponden a asignaturas que se desarrollan durante el primer y segundo semestre del respectivo año de estudio. Todas las asignaturas del currículo se desarrollan de forma semestral, por lo que la variable de rendimiento académico de las asignaturas seleccionadas contempló el promedio de notas finales del primer y segundo semestre, independiente del número de veces que un estudiante pudo haber cursado la asignatura (por reprobación u otras razones). Se consideró el promedio de nota final del primer intento en cada asignatura.

Análisis de datos. Se excluyeron los datos de alumnos que aún no hubiesen completado el programa de estudios. La planilla de datos fue exportada a los programas estadísticos SPSS $®$ versión 20 y AMOS $®$ versión 18 , donde se realizaron todos los análisis. La significancia se consideró con un valor inferior a 0,05 .

El análisis de datos se llevó a cabo en cuatro etapas. En primer lugar se calcularon los datos descriptivos, luego se realizó una comparación de diferencias de medias con respecto al sexo para cada variable de estudio. El resultado de esta diferencia de grupos determinó la inclusión del sexo como variable control en el modelo hipotetizado (Fig. 1). Esto se realizó mediante pruebas t de student para muestras independientes, para las cuales se calculó además el intervalo de confianza y el tamaño del efecto (Field, 2013). Posteriormente se calcularon las correlaciones mediante el coeficiente de correlación de Pearson.

Finalmente, mediante el análisis de ecuaciones es- 
GROB, M.; ORSINI, C.; BECERRA, D.; SABAG, N. \& ROJAS, M. P. El rendimiento académico de estudiantes de odontología en la asignatura de morfología como predictor del tiempo real de titulación y rendimiento académico en asignaturas de alto crédito. Int. J. Morphol., 38(5):1508-1512, 2020.

tructurales, se integraron las variables en un modelo (Fig. 1) para analizar el ajuste de éste a los datos y los recorridos de regresión entre las diferentes variables. El modelo se estimó mediante el método de máxima verosimilitud. La bondad de ajuste del modelo a las observaciones se calculó a través de una serie de estadísticos (Kline, 2010). Estos incluyeron (con su criterio de aceptación entre paréntesis) la prueba de Chi cuadrado (c2, >0,05), el goodness-of-fit index (GFI, >0,90), el comparative fit index (CFI, >0,90), y el root mean square error of approximation (RMSEA, <0,08). Los recorridos entre las variables fueron estimados e interpretados como coeficientes de regresión múltiple.

\section{RESULTADOS}

Luego de descartar datos de estudiantes que hayan desertado en algún momento de la carrera o aún no completado su programa de estudio, se procedió a analizar los resultados considerando los registros de 672 estudiantes entre las cohortes de ingreso 2002-2012. De estos, 486 (72 \%) correspondieron a mujeres y $186(28 \%)$ correspondieron a hombres.

Datos descriptivos y comparación de medias. La Tabla I muestra los datos descriptivos para todas las variables y la comparación de medias respecto al sexo. El mayor rendimiento académico (en escala de 1,0 a 7,0) se registra para la asignatura de CIA5, seguido por CIA4, Morfología, y finalmente PA. No obstante, todos los valores se encuentran por sobre el umbral de aprobación (4,0). El TRT correspondió a 6,60 años con respecto a los 6 años de titulación formal. Con respecto a las diferencias por sexo, no se reportaron diferencias estadísticamente significativas para el RAM, PA, y CIA4. Las mujeres presentaron un rendimiento académico significativamente superior en la asignatura de CIA5 y un TRT significativamente menor que los hombres. No obstante, el tamaño del efecto para estas diferencias fue pequeño.
Correlaciones. La Tabla II muestra las correlaciones entre todas las variables de estudio. Todas las correlaciones reportadas se muestran como significativas. El RAM mostró asociaciones positivas (débiles a moderadas) con todas las demás asignaturas en orden decreciente con respecto a su proximidad en el programa de estudio. Se reportó una correlación negativa moderada entre el RAM y el TRT ( $r=-$ 0,48 p<,01). Las asignaturas de PA y CIA4 y CIA5 también mostraron correlaciones positivas entre ellas y correlaciones negativas moderadas a fuertes con respecto al tiempo real de titulación.

Modelo de ecuaciones estructurales. Como primer paso se analizó el ajuste del modelo a los datos. Los estadísticos de bondad de ajuste mostraron que la prueba de Chi cuadrado fue significativa $(\mathrm{X} 2=8,762, \mathrm{df}=2, \mathrm{P}=0,013)$, lo que sugeriría un pobre ajuste. Por esto se utilizaron también estadísticos de ajuste menos sensibles al tamaño de la muestra, donde $\mathrm{GFI}=0,995, \mathrm{CFI}=0,993$ and $\mathrm{RMSEA}=0,07$ (CI, 0,03, 0,12 ) resultaron todos por sobre el estándar de aceptación, sugiriendo un adecuado ajuste.

El segundo paso consistió en calcular los coeficientes de regresión para el modelo. En la Figura 2 se muestra el modelo propuesto con sus valores estandarizados de regresión, donde todas las relaciones entre variables fueron significativas y en la dirección hipotetizada. El RAM predijo de forma positiva el rendimiento académico en las asignaturas de PA $(b=0,250$ $\mathrm{p}<, 001)$ y CIA4 $(\mathrm{b}=0,198 \mathrm{p}<, 001)$ y CIA5 $(\mathrm{b}=0,138$ $\mathrm{p}<, 001)$. Con respecto a la influencia del rendimiento académico en el TRT, se observó que tanto la relación directa del RAM ( $b=-0,258$ p<,001) como su influencia a través del rendimiento académico de las demás asignaturas predijo de forma negativa el TRT. Finalmente, se reporta que el coeficiente de determinación (R2) muestra que el modelo propuesto explica en un 68,5\% la varianza en el TRT, mientras que un $31,5 \%$ de la varianza de esta variable es explicada por factores externos al modelo.

Tabla I. Media (y desviación estándar) para el total de la muestra, mujeres y hombres, y diferencias por sexo.

\begin{tabular}{|c|c|c|c|c|c|c|c|c|}
\hline \multirow[b]{2}{*}{ V ariables } & \multicolumn{3}{|c|}{ Medias (DE) } & \multicolumn{5}{|c|}{ Diferencias por Sexo } \\
\hline & $\begin{array}{c}\text { Total } \\
(\mathrm{n}=672)\end{array}$ & $\begin{array}{c}\text { Mujeres } \\
(\mathrm{n}=486,72 \%)\end{array}$ & $\begin{array}{c}\text { Hombres } \\
(\mathrm{n}=186,28 \%)\end{array}$ & $\begin{array}{l}\text { Diferencia } \\
\text { de medias }\end{array}$ & IC $95 \%$ & $\mathrm{t}$ & $\mathrm{p}$ & $\begin{array}{l}\text { Tamaño } \\
\text { del Efecto }\end{array}$ \\
\hline RAM & $4,51(0,57)$ & $4,51(0,58)$ & $4,51(0,55)$ & 0,01 & {$[-0,09,0,10]$} & 0,11 & 0,912 & 0,01 \\
\hline RAP & $4,39(0,64)$ & $4,41(0,64)$ & $4,32(0,66)$ & 0,09 & {$[-0,01,0,21]$} & 1,80 & 0,073 & 0,15 \\
\hline RACI4 & $4,58(0,92)$ & $4,56(0,93)$ & $4,65(0,90)$ & $-0,09$ & {$[-0,25,0,06]$} & $-1,18$ & 0,238 & $-0,10$ \\
\hline RACI5 & $4,93(0,70)$ & $4,98(0,63)$ & $4,78(0,84)$ & 0,20 & {$[0,06,0,33]$} & 2,90 & 0,004 & 0,26 \\
\hline TRT & $6,60(0,76)$ & $6,55(0,72)$ & $6,70(0,83)$ & $-0,15$ & {$[-0.29,-0,01]$} & -2.17 & 0,030 & $-0,19$ \\
\hline
\end{tabular}

Nota: RAM: Rendimiento académico asignatura de Morfología, RAP: Rendimiento académico asignatura de Preclínico del Adulto, RACI4: Rendimiento académico asignatura de Clínica Integral del Adulto de 4to año, RACI5: Rendimiento académico asignatura de Clínica Integral del Adulto de 5to año, TRT: Tiempo real de titulación, IC $95 \%$ : Intervalo de Confianza al $95 \%$, Tamaño del Efecto calculado en base a la d de Cohen $(<0,2=$ efecto pequeño, $0,5=$ efecto mediado, $>0,8=$ efecto grande). 


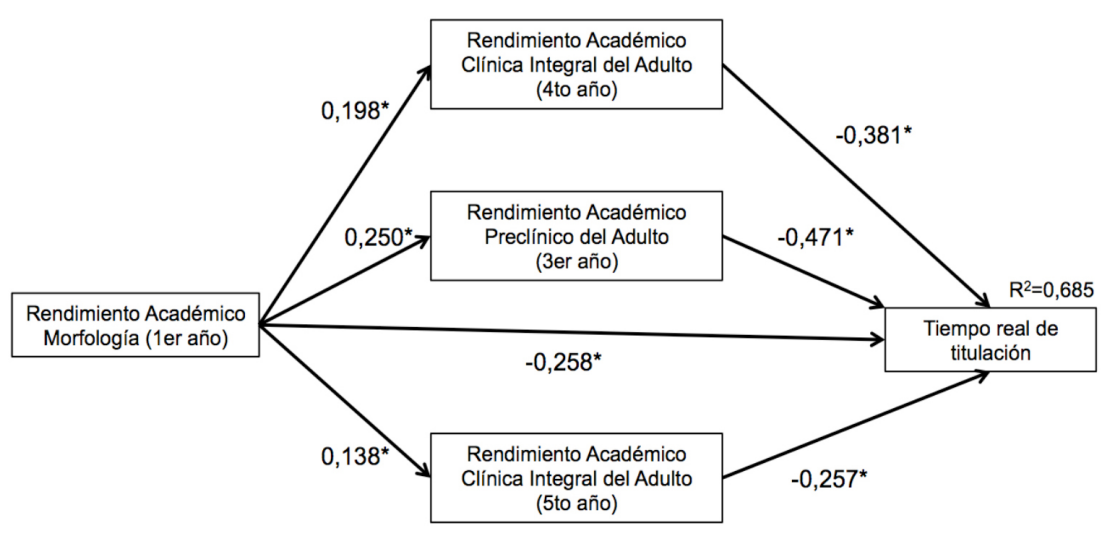

Fig. 2. Modelo de ecuaciones estructurales mostrando los coeficientes estandarizados de regresión entre las variables estudiadas. Nota: Residuales y covarianzas han sido omitidos para simplificar la visualización del modelo. La significancia está basada en los coeficientes no estandarizados. R2: coeficiente de determinación, ${ }^{*} \mathrm{p}<0,001$ taca como predictor del rendimiento académico básico y preclínico en estudiantes de medicina, a las estrategias de aprendizaje en interacción con otras variables individuales como sexo y otras como los métodos de evaluación. Por otro lado, Woods et al. establece que los médicos son más precisos en sus diagnósticos al tener conocimiento de las ciencias básicas. Sin embargo, no hay estudios realizados en estudiantes de Odontología que tomen específicamente a la asignatura de Morfología como factor predictor del rendimiento en las asignaturas Preclínica y Clínica, ni menos como predictor del TRT. Por lo mismo, este estudio resulta ser un aporte y un 'pun-
Tabla II. Correlaciones bivariadas entre las variables de estudio.

\begin{tabular}{lccccc}
\hline & RAM & RAP & RACI4 & RACI5 & TRT \\
\hline RAM & - & $0,25 * *$ & $0,20 * *$ & $0,14 * *$ & $-0,48 * *$ \\
RAP & & - & $0,15 * *$ & $0,18^{* *}$ & $-0,63 * *$ \\
RACI4 & & & - & $0,09 *$ & $-0,51 * *$ \\
RACI5 & & & & - & $-0,40 * *$ \\
TRT & & & & & - \\
\hline
\end{tabular}

Nota: RAM: Rendimiento académico asignatura de Morfología, RAP: Rendimiento académico asignatura de Preclínico del Adulto, RACI4: Rendimiento académico asignatura de Clínica Integral del Adulto de 4to año, RACI5: Rendimiento académico asignatura de Clínica Integral del Adulto de 5to año, TRT: Tiempo real de titulación. * $\mathrm{p}<0,05$ (bilateral), $* * \mathrm{p}<$ 0,01 (bilateral).

\section{DISCUSIÓN}

De acuerdo a nuestros resultados, se observan asociaciones que confirman nuestra primera hipótesis entre RAM y rendimiento de PA y CIA4 y CIA5. A medida que el resultado obtenido por los estudiantes en el primer intento al cursar la asignatura de Morfología aumentó, esto se asoció a un aumento en el rendimiento académico al cursar las demás asignaturas en su primer intento, por lo que el RAM puede ser tomado como predictor sobre el rendimiento de las asignaturas de PA y CIA4 y CIA5. La asociación fue mayor con la asignatura de PA y menor con las asignaturas de CIA4 y CIA5. Esto podría tener una explicación en la cercanía en que se dictan ambos cursos como a su vez en otros factores inherentes a la atención de pacientes propios de los ramos Clínicos.

Estudios anteriores concuerdan que el dominio de las ciencias básicas es un elemento predictor en el rendimiento académico. En el área de la medicina, Zúñiga des- to de partida' para futuros estudios que busquen indagar sobre factores que influyan directamente sobre el tiempo de titulación y el rendimiento en las asignaturas de alto crédito.

De acuerdo a nuestra segunda hipótesis, los resultados obtenidos nos muestran una asociación negativa moderada entre el rendimiento de Morfología y el TRT. Es decir, a medida que el RAM en el primer intento disminuye, esto se asocia con un aumento en el TRT de los estudiantes, siendo esta asociación tanto de forma directa como indirecta a través de las asignaturas de PA y CIA4 y CIA5. Estos resultado son de particular relevancia ya que reflejan la importancia en el rendimiento académico y experiencia que tengan los estudiantes al cursar por primera vez la asignatura de Morfología, lo cual podría influir sobre su futuro desarrollo y rendimiento predictor en el plan de estudio y en el tiempo que requerirían para completarlo de forma satisfactoria.

Este estudio nos permite tener una visión sobre la relación que existe entre las asignaturas de ciencias básicas como predictores sobre el rendimiento de asignaturas clínicas fundamentales y sobre el TRT, tomando en cuenta que muchos estudios anteriores se han enfocado más en estudiar otros predictores, como el rendimiento académico en la etapa escolar, o los resultados de las pruebas de admisión (Mitchell, 1990; Zúñiga et al.). De esta forma, es posible identificar a aquellos alumnos que podrían tener dificultades para cursar asignaturas en el transcurso de la carrera, y ofrecer orientación para culminar los estudios en el tiempo adecuado. Esto permite brindar herramientas de apoyo para cursar la carrera de manera óptima, mejorar la experiencia universitaria de los estudiantes y la calidad de los futuros odontólogos. 
GROB, M.; ORSINI, C.; BECERRA, D.; SABAG, N. \& ROJAS, M. P. El rendimiento académico de estudiantes de odontología en la asignatura de morfología como predictor del tiempo real de titulación y rendimiento académico en asignaturas de alto crédito. Int. J. Morphol., 38(5):1508-1512, 2020.

No obstante, los resultados del presente estudio deben ser interpretados con precaución considerando sus limitaciones. Por una parte, sería importante complementar el modelo de rendimiento académico con otras variables que podrían influir en el TRT y examinar cuan generalizable es en otros contextos universitarios. Por otra parte, aún cuando el modelo tiene un valor de correlación alto con el TRT, se necesitan más estudios para evaluar en que medida se ve afectado este ratio. Para futuras investigaciones sería interesante indagar sobre los métodos de evaluación, ya que al saber con más certeza el área o método evaluador de la asignatura que impacta mayormente en el rendimiento de ellos, se puede proporcionar un apoyo o ayuda más personalizada y eficaz.

Los resultados de este estudio contribuirán a robustecer la evidencia disponible en relación a la relevancia del estudio y buen desempeño en Morfología para el desarrollo y formación de los estudiantes a lo largo del currículo Odontológico, además de justificar la identificación oportuna y apoyo para aquellos estudiantes que estén experimentando dificultades en esta asignatura.

AGRADECIMIENTOS. Se agradece la colaboración de Sra. Victoria Riumalló en la recolección de datos.

GROB, M.; ORSINI, C.; BECERRA, D.; SABAG, N. \& ROJAS, M. P. Academic performance of dental students in the subject of morphology as a predictor of their actual graduation time and academic performance in high credit subjects. Int. J. Morphol., 38(5):1508-1512, 2020.

SUMMARY: The relevance of basic sciences as predictors of students' academic performance has great impact in healthcare education. Therefore, the aim of this study was to analyze the predictive role of dental students' academic performance in the subject of Morphology on high credit subjects in the preclinical and clinical phase, and on real time of graduation. A retrospective correlational study was conducted with 672 dental students (2002 to 2012) at the Universidad de los Andes, Chile. The variables studied were sex, year of admission and graduation, and academic performance in the subjects of Morphology (first year), Preclinics (third year), and Integral Adult Clinics of fourth and fifth year. Data analysis were conducted by means of Pearson's correlation tests and structural equation modeling. Morphology academic performance was significantly associated, and predicted the academic performance in Adult Preclinical $(r=0.25 \mathrm{p}<.01, b=0.250 \mathrm{p}<.001)$, fourth-year Adult Clinic $(r=0.20 \mathrm{p}<.01$, $\mathrm{b}=0.198 \mathrm{p}<.001)$ and fifth-year Adult Clinic $(\mathrm{r}=0.14 \mathrm{p}<.01, \mathrm{~b}=0.138$ $\mathrm{p}<.001$ ), and was negatively with real graduation time. The model analyzed explained a $68.5 \%$ variance in real time of the graduation. The results of this study contribute to strengthening the available evidence in relation to the relevance of students' performance in the subject of Morphology for their development and training throughout the dental curriculum, as well as justifying the timely identification and support for those students experiencing difficulties in this subject.

KEY WORDS: Actual graduation time; Credit transfer system; Dentistry; Higher education; Morphology.

\section{REFERENCIAS BIBLIOGRÁFICAS}

ADEA Foundation Knowledge and Skills for the New General Dentist: (As approved by the 2011 ADEA House of Delegates). J. Dent. Educ., 81(7):848-52, 2017.

Australian Dental Council. Professional competencies of the newly qualified dentist. Melbourne, Australian Dental Council, 2016.

Bennett, J. \& Beeley, J. A. ADEE meetings focus on the basic sciences: meeting reports. Eur. J. Dent. Educ., 17(2):72, 2013.

Fiehn, N. E. The basic science teaching experience in the Nordic countries. Eur. J. Dent. Educ., 2(3):115-23, 1998

Field, A. Discovering Statistics Using IBM SPSS Statistics. London, Sage, 2013

Field, J. C.; Cowpe, J. G. \& Walmsley, A. D. The graduating European dentist: a new undergraduate curriculum framework. Eur. J. Dent. Educ., 21 Suppl. 1:2-10, 2017.

Grob, M.; Becerra, D.; Rodriguez, A.; Cristiane, J.; Ramirez, V. \& Sabag, N. Relationship between university selection test scores, high school grade point average (GPA) and academic performance in the subject of morphology in dentistry freshmen at the Universidad de los Andes. Int. J. Morphol., 33(2):527-31, 2015.

Hassan, S. Concepts of vertical and horizontal integration as an approach to integrated curriculum. Educ. Med. J., 5(4):e1-5, 2013.

Jerez, O.; Orsini, C.; Hasbún, B.; Lobos, E. \& Muñoz, M. Is undergraduate programme accreditation influenced by educational public policy quality indicators? An exploratory study of the Chilean higher education quality assurance system. High. Educ. Policy, 31:121-38, 2018.

Kline, R. B. Principles and Practice of Structural Equation Modeling. New York, Guilford Publications, 2010.

Martínez-Alvarez, C.; Sanz, M. \& Berthold, P. Basic sciences education in the dental curriculum in Southern Europe. Eur. J. Dent. Educ., 5(2):63-6, 2001.

Mitchell, K. J. Traditional predictors of performance in medical school. Acad. Med., 65(3):149-58, 1990.

Plasschaert, A. J.; Lindh, C.; McLoughlin, J.; Manogue, M.; Murtomaa, H.; Nattestad, A. \& Sanz, M. Curriculum structure and the European Credit Transfer System for European dental schools: part I. Eur. J. Dent. Educ., 10(3):123-30, 2006

Rodríguez Fontes, R.; Bacallao Gallestey, J.; Díaz Rodríguez, P. A. \& Morejón Lazo, M. Valor predictivo de algunos criterios de selección para el ingreso a la carrera de medicina. Educ. Med. Super, 14(1):17-25, 2000

Serrano, C. M.; Botelho, M. G.; Wesselink, P. R. \& Vervoorn, J. M. Challenges in the transition to clinical training in dentistry: An ADEE special interest group initial report. Eur. J. Dent. Educ., 22(3):e451-e457, 2018.

Woods, N. N.; Brooks, L. R. \& Norman, G. R. The value of basic science in clinical diagnosis: creating coherence among signs and symptoms. Med. Educ., 39(1):107-12, 2005.

Zúñiga, D.; Mena, B.; Oliva, R.; Pedrals, N.; Padilla, O. \& Bitran, M. Modeling the academic performance of medical students in basic sciences and preclinical courses: a longitudinal study. Rev. Med. Chile, 37(10):1291300,2009 .

Dirección para correspondencia:

Melisa Grob

Unidad de Morfología

Facultad de Odontología

Universidad de los Andes

Mons. Álvaro del Portillo 12.455

Las Condes

Santiago - CHILE

Email: mgrob@uandes.cl

Recibido : 04-03-2020

Aceptado: 30-04-2020 